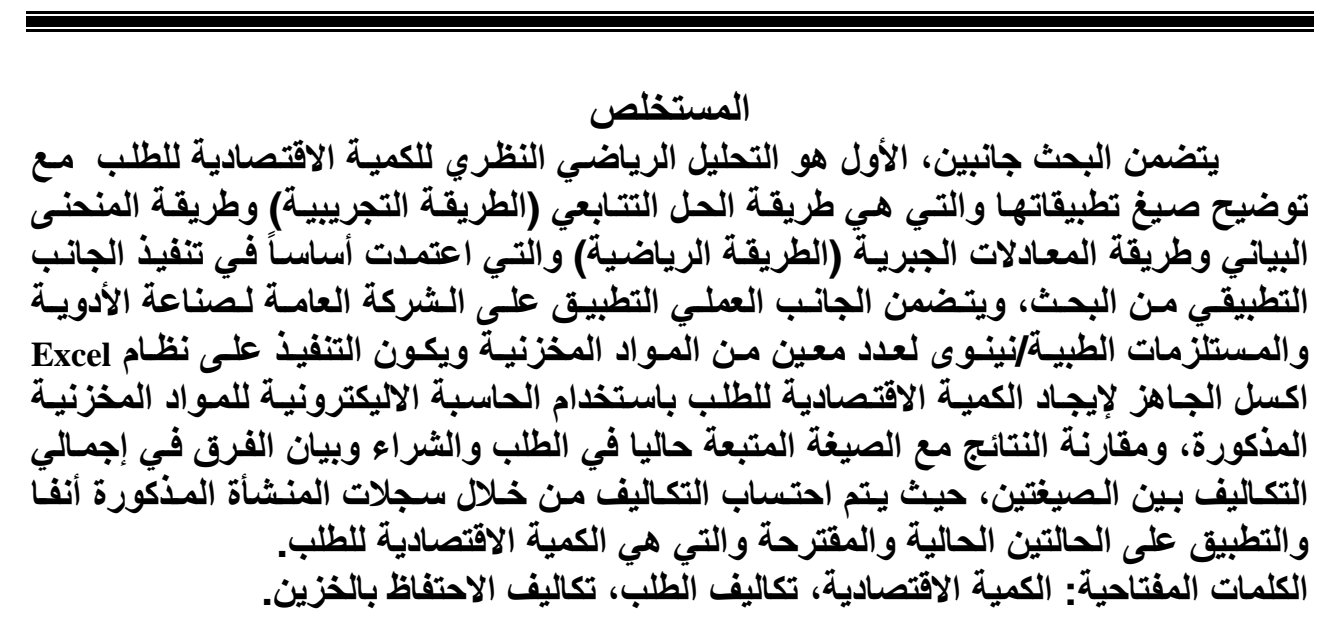

\title{
Managing the Economic Order Quantity: A Case Study
}

\author{
Ayad A. Abdul Kadir \\ Lecturer \\ Department of Industrial Management \\ University of Mosul \\ ayad_khattab@yahoo.com
}

\begin{abstract}
The research includes two parts; the first is the theoretical mathematical analysis of the economic order quantity with its types of application. It includes the sequential solving method (the experimental method), the graphic curve method, and the algebra equation method (the mathematical method) which was used as the basis of carrying out the application part of the research. The practical part includes application at Nineveh Drug

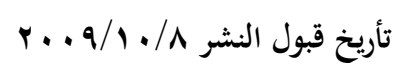

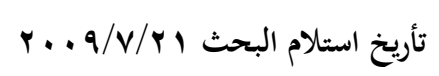


Industry and Medical Appliances/Nineveh (NDI) on a certain number of storage items and the application is to be carried out by MS Excel to calculate the economic order quantity by using computer to calculate the previously mentioned storage items, comparing the results and outcomes with those of the method currently used for ordering and buying and demonstrating the difference in total costs between both methods. Costs are calculated by the above-mentioned industry's archive files. The application is carried out for both methods, current and suggested the economic order quantity method.

Key Words: Economic Order Quantity, (Ordering Costs), Holding Costs.

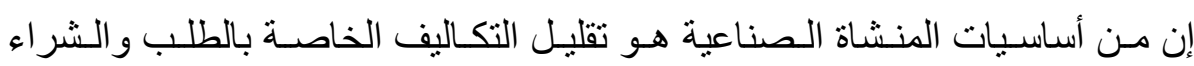

و الخزن وتحديد الاحتياجـات من المـواد الاحتياطيـة من حيـث أوصسافها وكمياتها ومو اعيد الحاجة إليها ومصادر وظروف تجعيز ها وبأقل كلفة اقتصادية مدكنة وذللك باعتمـاد استخدام

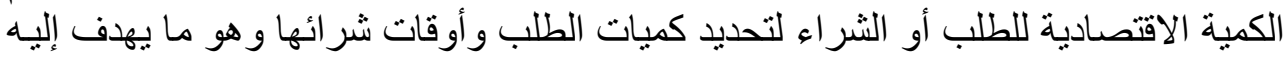
البحث الحالي.

أولاًا منهجية البحث

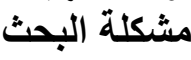

تتحدد مشكلة البحث بعدم اهتمام معظم المنشآت الصناعية بالأسـاليب العلميـة لتخطيط

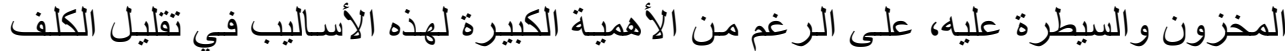

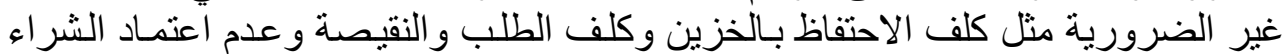

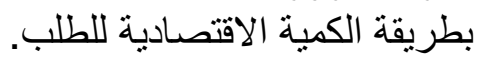

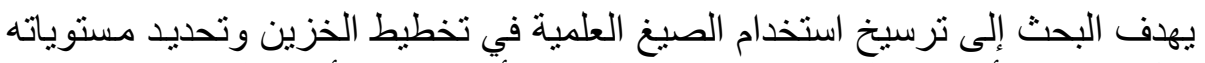
هدف البحث

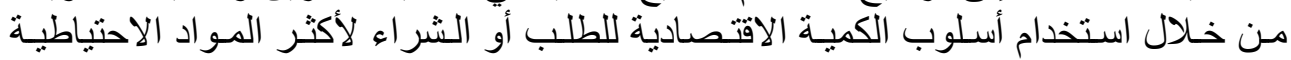
حركة في مخازن المنشأة الصناعية.

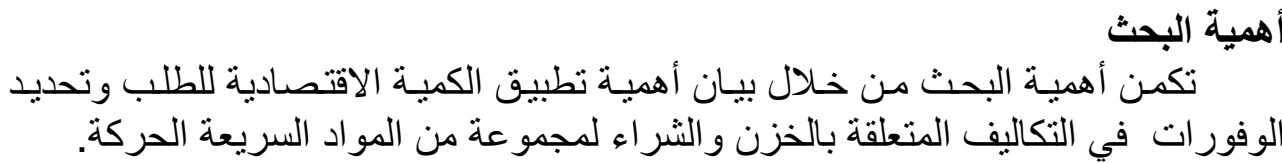
فرضية البحث

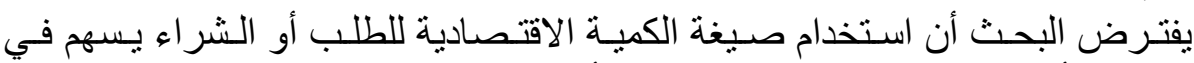
تخفيض الكلف أكثر من الاستخدام الحالي للطلب أو الشراء. أسلوب جمع البيانات

اعتمد البحث على الأسلوب الاستقرائي في بلورة الجانب النظري إضـافة إلى استخدام البيانات والأرقام من و اقع سجلات الثركة المبحوثنة في تدعيم الجانب المبل المبداني. الإجراءات المتبعة في البحث فن أنع 


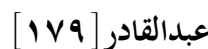

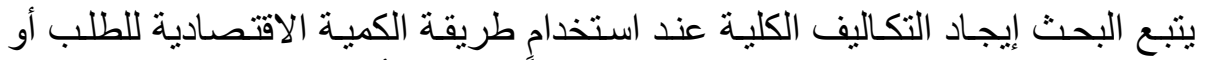

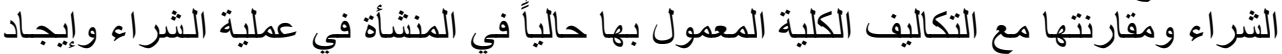

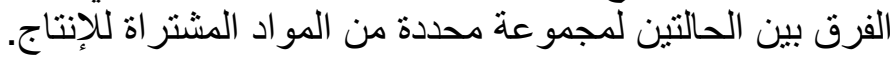

دية للاطب

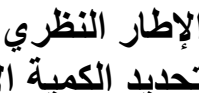

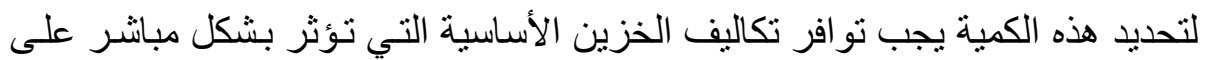

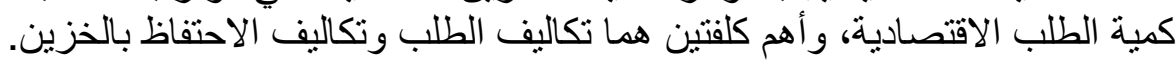

1. تكاليف الطلب (Ordering Costs)

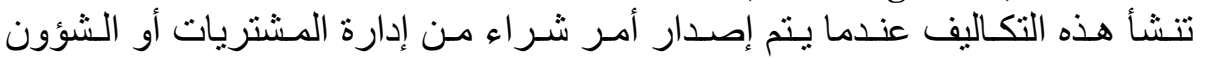

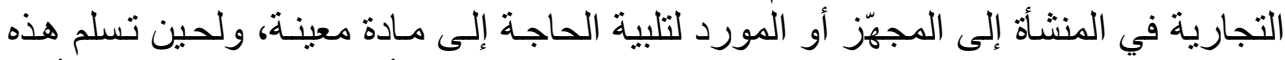

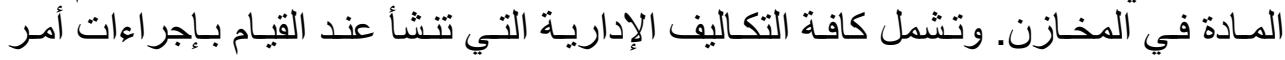

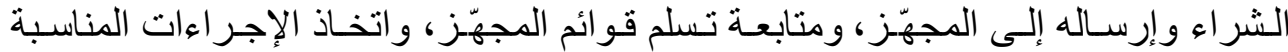

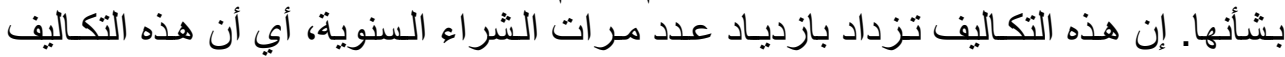

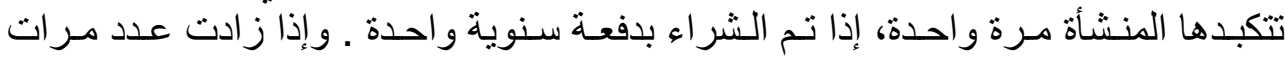

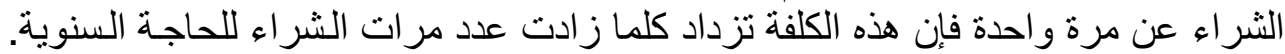

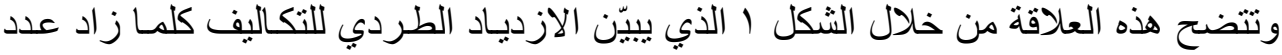

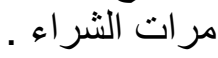

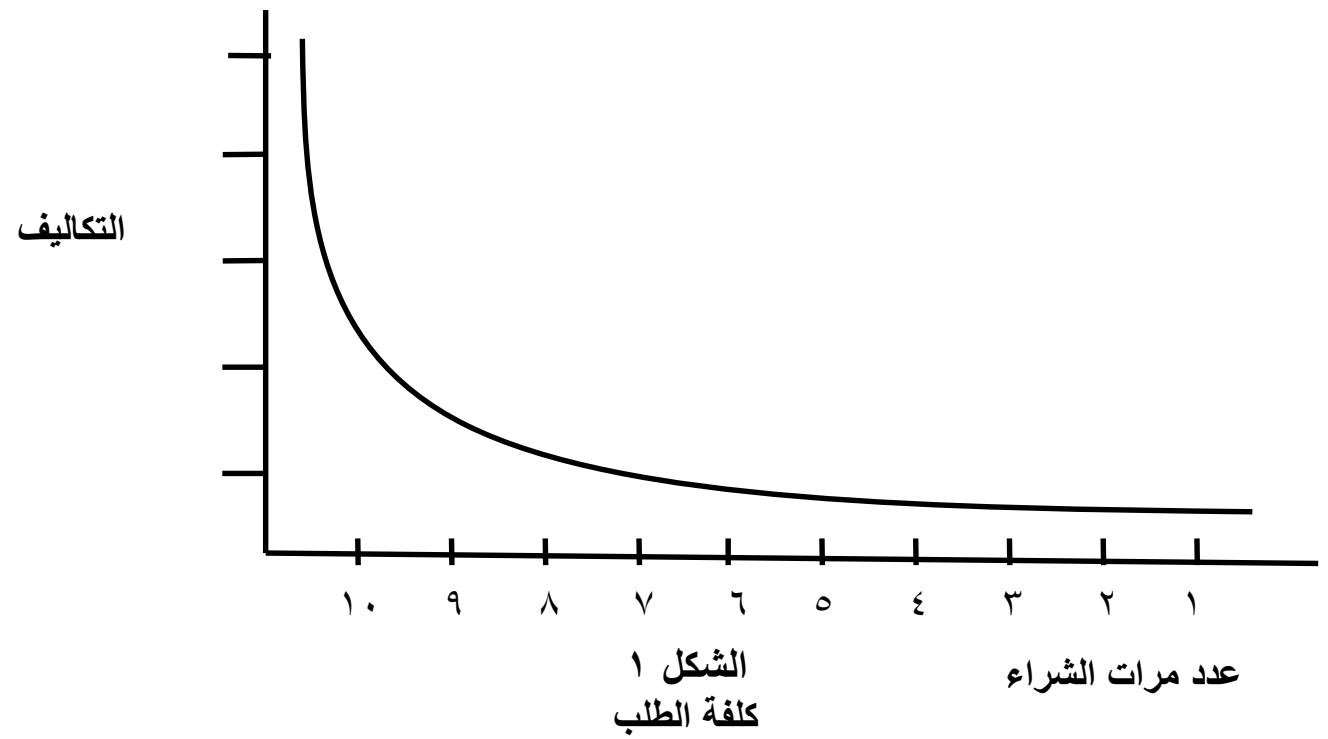

المصدر: جاسـم ناصـر حسين وآخرون، الأصسول العلميـة في تخطبط ورقابـة المخزون، مطسابع

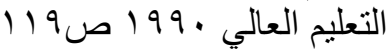


ا ـ تكاليف الطلب الواحد من المصاريف الإداريـة لإدارة المشتريات المصروفة على تهيئة

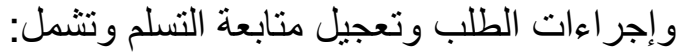

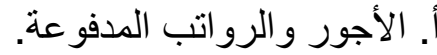
ب. ب. إيجار أو (اندثار) الأبنية. ت. ت. اندثار الأجهزة و المعدات والاتهة والأثاث.

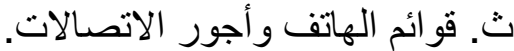
ج. قيمة القرطاسية وكافة المواد الماد المستهلكة الأخرى. ح. مصاريف الضيافة و المكافآت و الهدايا. خ. كافة المصاريف المشابهة الأخرى.

$$
\begin{aligned}
& \text { r. تكاليف النقل و التقريغ. } \\
& \text { r. بـ تكاليف الاستلام. } \\
& \text { ع ـ تكاليف فحص الجودة. }
\end{aligned}
$$

r. rolding Costs) تكاليف الاحتفاظ بالخزين و هي مجموعة التكاليف التي تتحملها المنشأة عند تخزين كميـة من المواد أو السلع وتتكون من العناصر الآتية: ا ـ تكاليف استثمار راس المال بالخزين (وكلفة الفرصة البديلة).

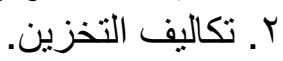
r. ع. ت تكاليف التلف.

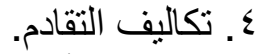

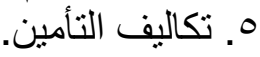

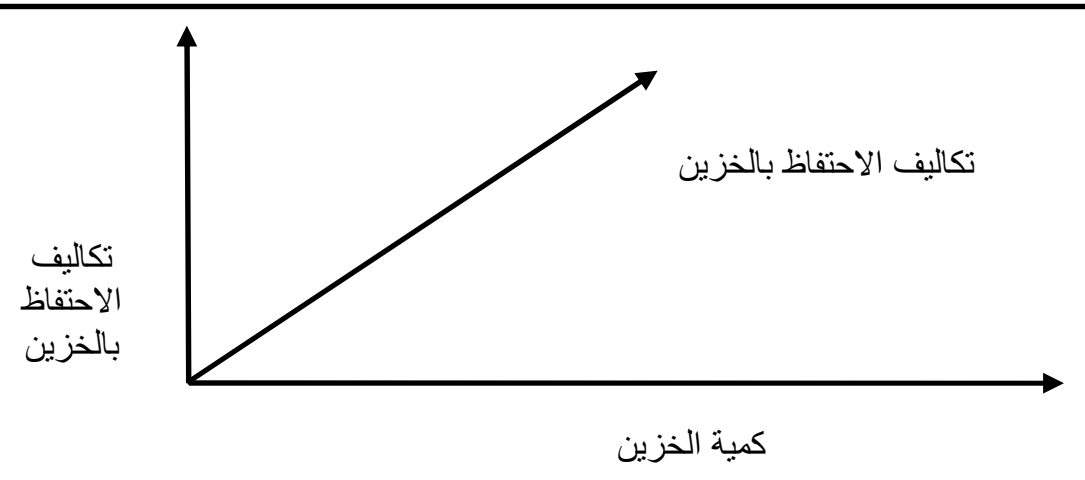

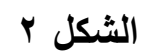
كلقة الخزين

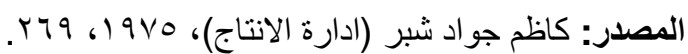


عبدالقادر[11

تتكون من عناصر مختلفة من التكاليف وتتشأ من نفاد الخزين لمادة مخزنيـة معينة أو مجموعة مو اد والتي تؤثر في الإنتاج ومن هذن منه العناصر:

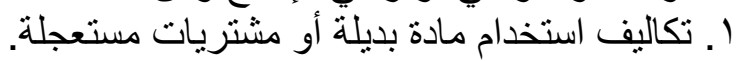

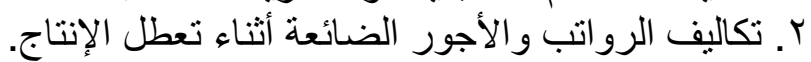
r. ب. تكاليف إعداد الآلات لإنتاج منتج آخر . ع. تكاليف المبيعات المفقودة.

ه. تكاليف الجهود الاستثنائية التي تدفع لتعجيل تجهيز المادئ التهادة النافدة.

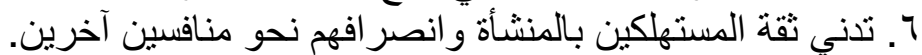

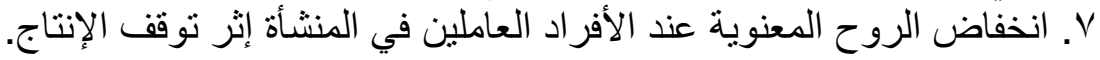

الكمية الاقتصادية للشراء

هي تلك الكميـة التي تتواءوازن أو تتساوى عندها تكاليف الطلب وتكـاليف الاحتفـاظ بالخزين السنوي. وتتخفض معها التكاليف الكلية السنوية إلى أدنى حد ممكن.

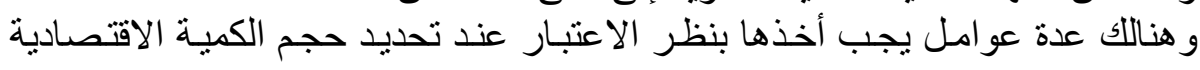
اللشر اء منها:

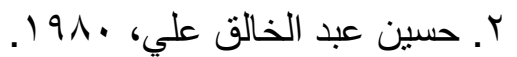

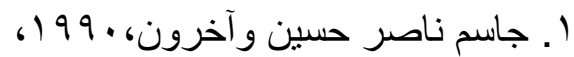

1 ا ـ معدلات الاستهلاك للمو اد في الفترات السابقة ومدى تذبذبها. Y. Y. مدة الانتظار أو الزمن القيادي (L.T.). ب. المساحات المخزنية وطاقاتها الاستيعابية.

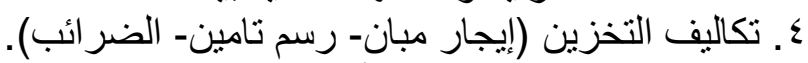

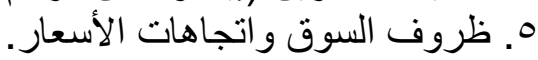
7. V. الكلفة الثابتة للطلبية الواحدة.

يتضح من الرسم البياني أن مسقط الحد الأدنى يمر بنقطة تقاطع منحنى كلفة الخزن مع تكاليف الطلب. 


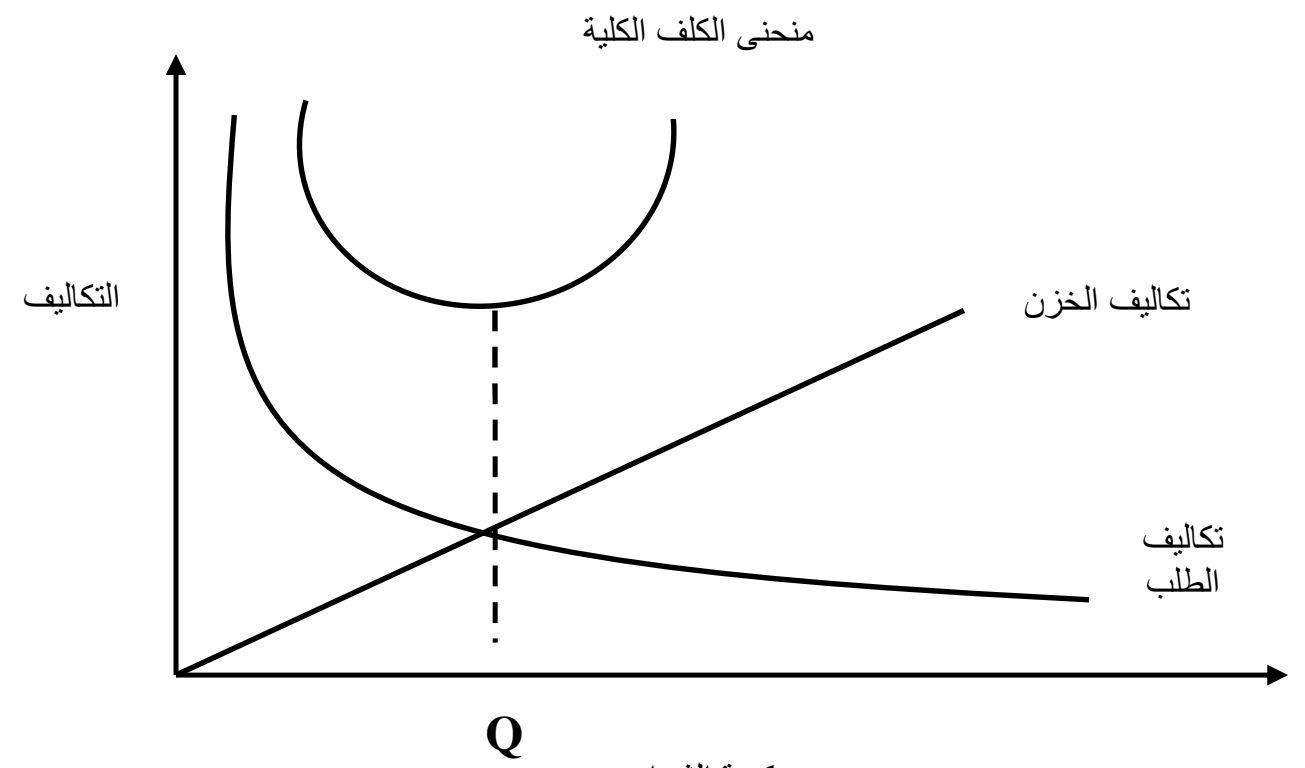

\section{Q

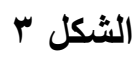

التكاليف الكلية للخزين

المصدر: حسين عبد الخالق علي (دراسة في الإنتاجية وعلاقتها بإدارة الخزين في المنشآت الصناعية)، 11 ₹ 6 191.

طرائق احتساب الكمبة الاقتصادية للثرائ

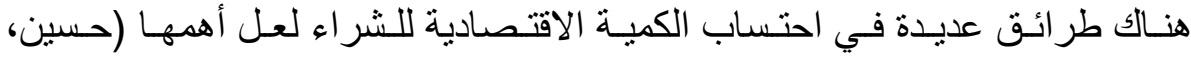
:(1)96)99.

\section{ا ـ طريقة الحل التتابعي (الطريقة التجريبية)}

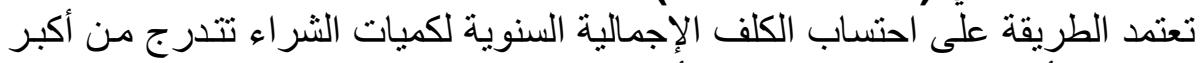

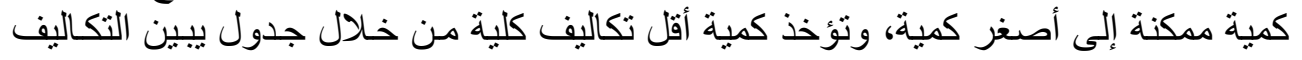
الكلية لكافة الكميات الممكنة.

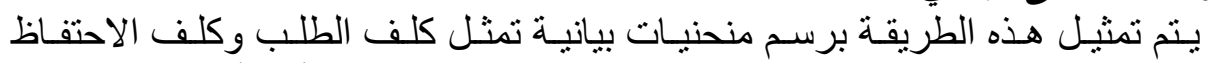

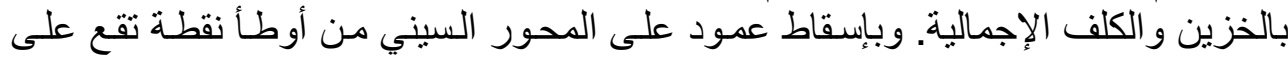

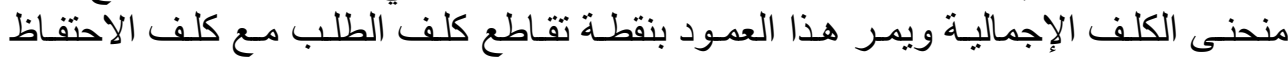

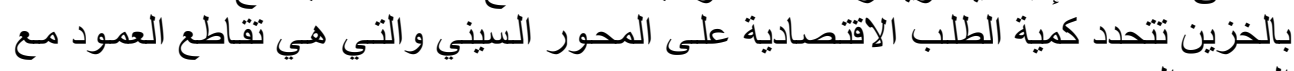
المحور السيني. 
r. طريقة المعادلات الجبرية (الطريقة الرياضية)

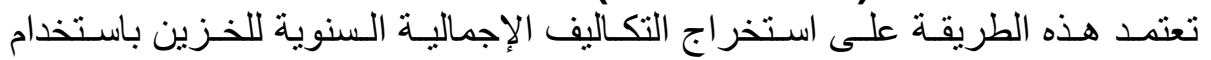

الدعادلات الرياضية للكلف الدكونة للتكاليف الإجمالية:

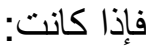

Tc = D سعر الوحدة الواحدة من المادة. C C كمية الثر اء الاقتصادية. $=$ Q Q

= Ch = R فإن معادلة التكاليف الكلية السنوية للمادة تكون:

الكلفة الإجماليـة السنوية للخزين = كلفة شـراء الكسادة السنوية + كلفة الاحتفـاظ بـالخزين

$$
\begin{aligned}
\therefore \quad \mathrm{Tc} & =\mathrm{DC}+\frac{Q}{2} C h+ \\
\frac{d T c}{d Q} & =\frac{C h}{2}+\left(-\frac{D R}{Q^{2}}\right) \\
= & \frac{C h}{2}=\frac{D R}{Q^{2}}
\end{aligned}
$$

السنوية + كلفة الطلبات السنوية (كلفة الثراء).

وللحصول على نقطة الحد الأدنى للتكاليف الكلية نساوي قيمة تفاضل الدالة الأولى لهى

$$
\begin{aligned}
& \frac{C h}{2}-\frac{D R}{Q^{2}}=0 \\
& \therefore \frac{C h}{2}=\frac{D R}{Q^{2}} \\
& Q^{2}=\frac{2 D R}{C h} \Rightarrow Q=\sqrt{\frac{2 D R}{C h}} \quad \text { وفي حالة توازن الكلف أو تساويها يكون: }
\end{aligned}
$$$$
\frac{Q}{2} C h=\frac{D}{Q} R
$$ 
$Q^{2}=\frac{2 D R}{C h}=Q=\sqrt{\frac{2 D R}{C h}}$

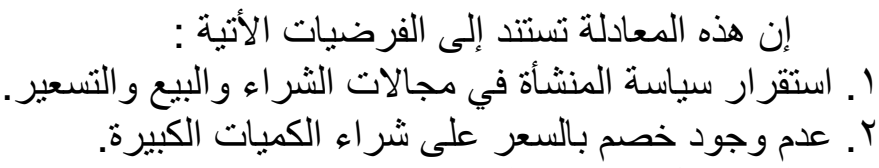
r. ب. تكاليف النقل ثنابتة مع الطلبية الواحدة.

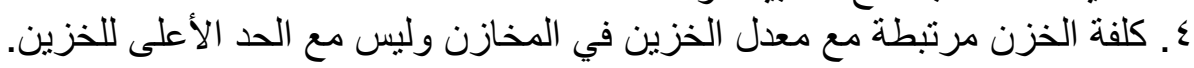

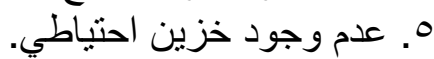

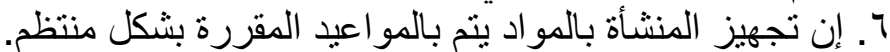

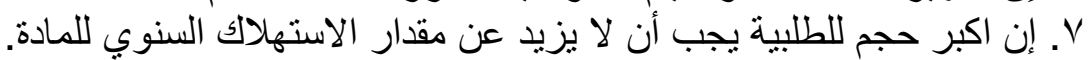

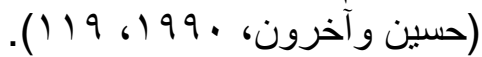

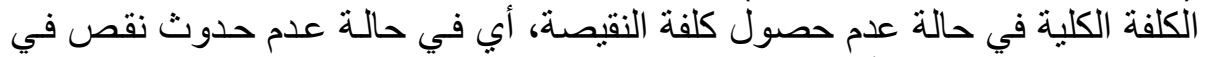

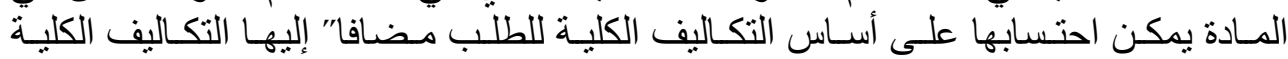
للاحتفاظ بالخزين وتكون على النحو الأتي (Slack, 2004, 418):

كلف الاحتفاظ بالخزين = كلفة خزن الوحدة الواحدة من المادة × معدل الخزين $\frac{Q}{2} \times C h=$ كلفة الاحتفاظ بالخزين وتكاليف الطلب = كلفة الطلب × عدد الطلبات في الفترة الزمنية الواحدة

$$
\begin{aligned}
& R \times \frac{D}{Q}=\text { =كاليف الطلب } \\
& \frac{R D}{Q}+\frac{C h Q}{2}=\frac{}{2} \\
& \frac{R D}{Q}+\frac{C h Q}{2}=\mathrm{Tc} \therefore
\end{aligned}
$$

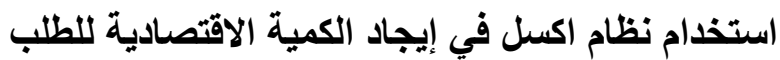

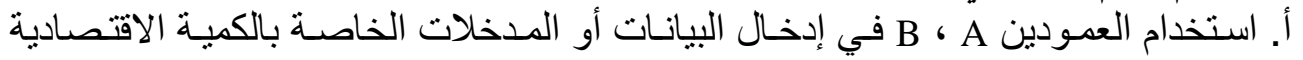

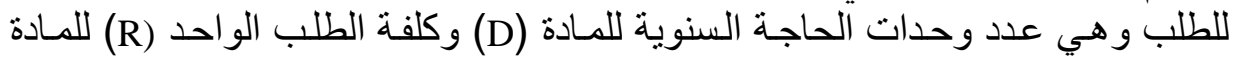

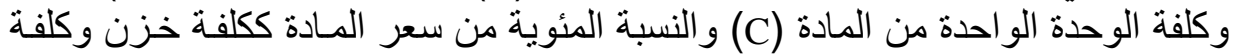

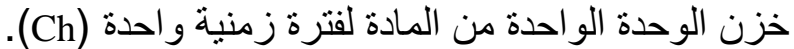
ب. استخدام العامودين E D ل إظظهار النتائج (Evans, 2004,507). 
عبدالقادر110]

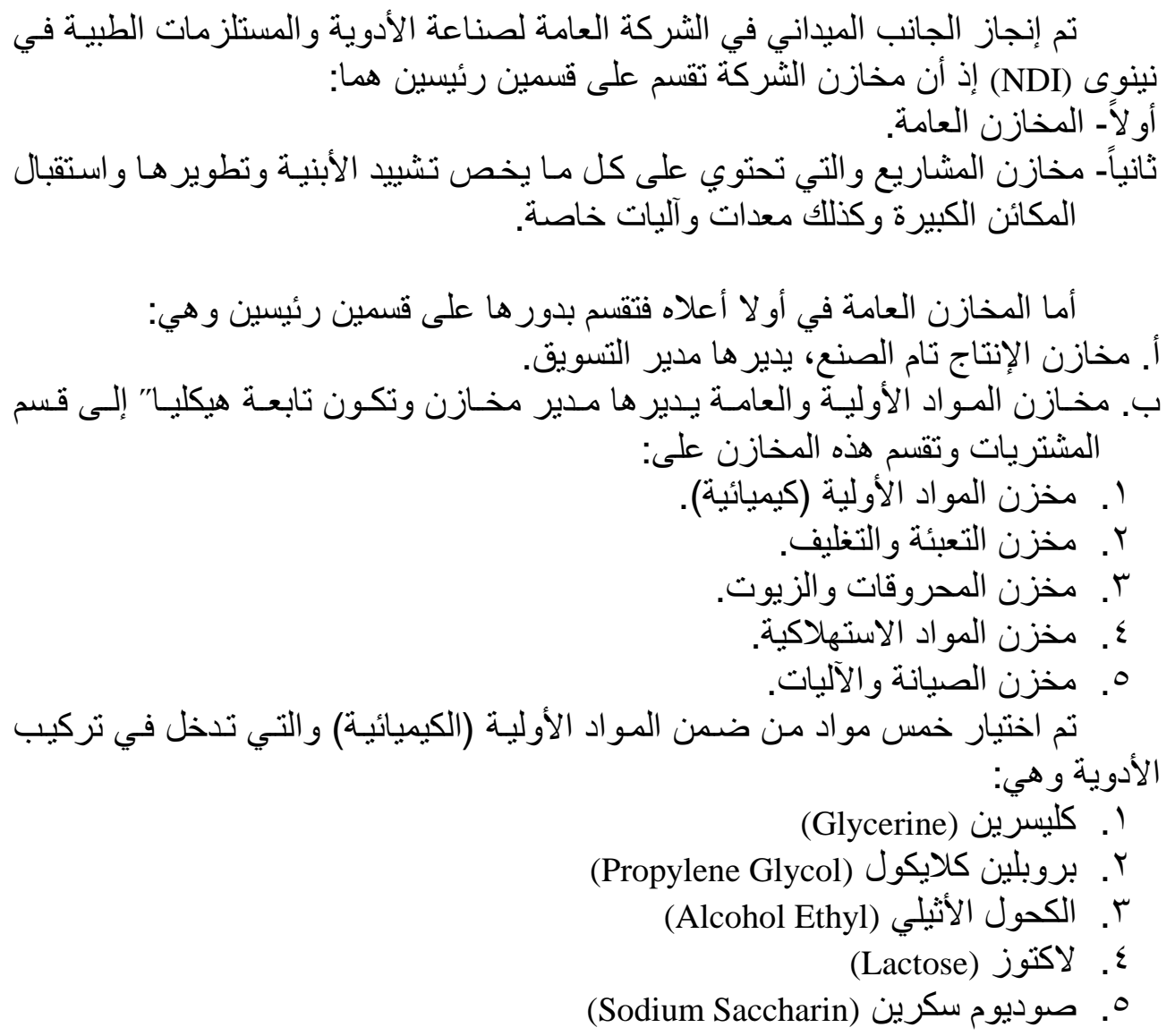




$$
\begin{aligned}
& \text { كمية الطلب الو احد حالياً ـ = . . . . . كغم للطلبية الواحدة } \\
& \frac{7 . . .}{r} \times \text { كلفة الخزن } \\
& 110 \wedge 9 \ldots=
\end{aligned}
$$

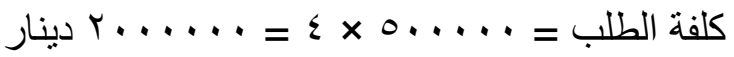

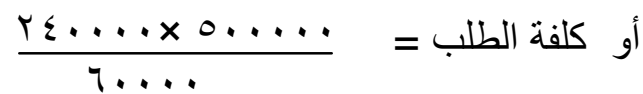

$$
\begin{aligned}
& \text {.... = }
\end{aligned}
$$

الكلفة الكلية = = 11019

1 1 دينار = . . =

$$
\text { ع.O.Q حالة استخدام }
$$

$$
\sqrt{\frac{500000 \times 240000 \times 2}{386.2}}=\sqrt{\frac{2 D R}{C h}}=Q
$$

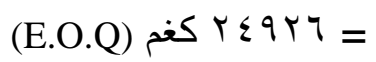

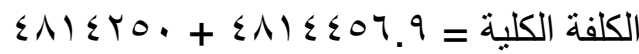

\begin{tabular}{|c|c|c|c|}
\hline الفرق & 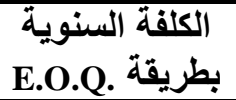 & الكلفة السنوية في في & اسم المادة \\
\hline
\end{tabular}
$97 r \wedge V \cdot V=$

أي بفارق . . . . . . . دينار. . . . . . و الصيغة نفسها بالنسبة لباقي المواد. دون.

\section{الجدول}

الفروقات بين الطريقة المتبعة حاليا وصيغة الكمية الاقتصادية للطلب على أساس الكلف الإلف الإجمالية للمواد الخمسة 


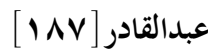

\begin{tabular}{|c|c|c|c|}
\hline الفرق & بطريقة الكلفة السنوية & الكلفة السنوية في & اسم المادة \\
\hline$r 97.49 r$ & QTYAV.V & $1 r 0 \wedge 9 \ldots$ & ا . كليسرين \\
\hline YqะqVqV & $r V \cdot r V \cdot r$ & $07010 .$. & r. بروبلين كلايكول \\
\hline rर9010 & 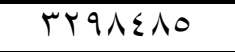 & roגᄉ... & ". الكحول الأثيلي \\
\hline $1 . \leqslant 01$ & $T r \leqslant \Lambda \cdot V T$ & TrONOTE & ع. لاكتوز \\
\hline $7010 \mathrm{~V}$ & rrVIrqr & YETVYO. & ○. صوديوم سكرين \\
\hline VYVO9Y. & & & إجمالي الفروقات \\
\hline
\end{tabular}

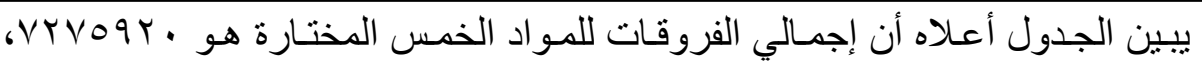

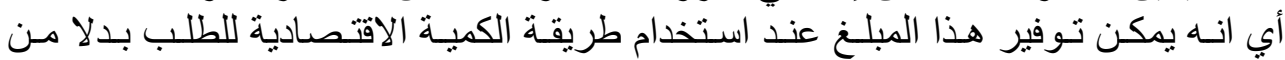

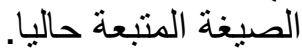

\section{الاستنتاجات والمقترحات}

الاستتناجات

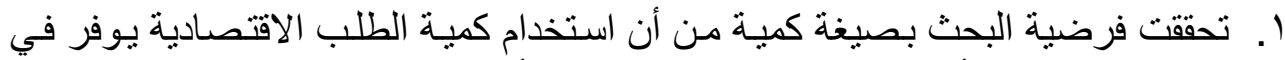

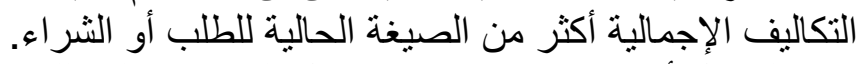

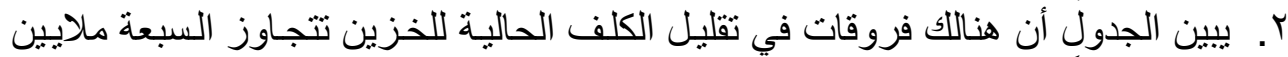
دينار سنوياً لخمس مو اد فقط.

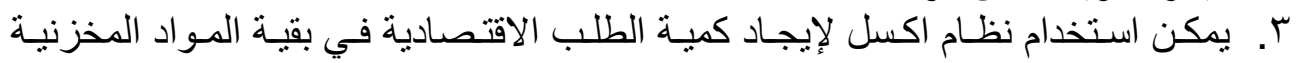
بصيغة استخدامه بالمواد الخمس المختارة نفسها.

\section{المقترحات}

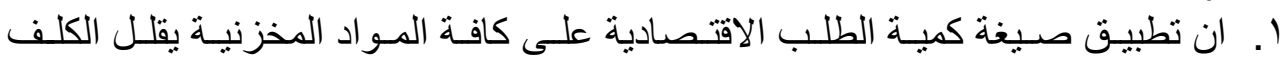
المخزنية بشكل كبير. r. . من المفضل استخدام نظلام اكسل على الحاسوب لتسهيل احتساب الكميـات و التكاليف لكافة المواد المخزنية. r. استخدام دالة الجذر التربيعي (SQRT) في نظام اكسل لإيجاد الكميـة الاقتصادية للطلب لكل مادة مخزنية. ع. . استخدام معادلة التكاليف الإجمالية في نظام اكسل لحساب الكلفة الإجمالية لكل مـادة ثم

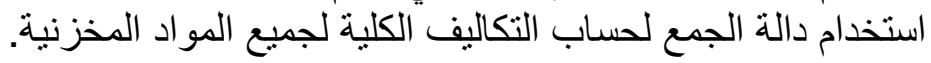




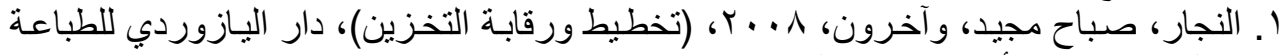
و النشر - عمان الأردن- شارع وآخرون الملا حسين.

r. حسين، جاسم ناصر، و آخرون، ـ 99 19، (الأصول العلمية في تخطيط ورقابة المخزون) مطابع

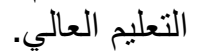

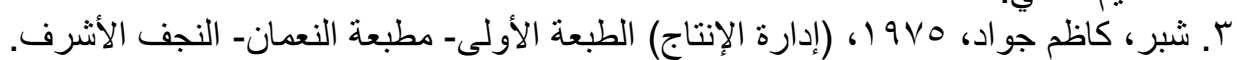

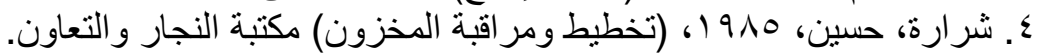

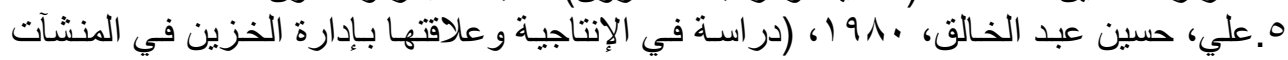

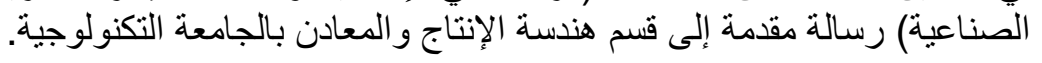

ثانياً المراجع باللغة الاجنبية

1. Evans, James R.; Collier, David A. 2007; (Evans/Collier) (Operations Management An Integrated Goods and Services Approach) \{Thomson- South-Western .

2. Slack, Nigel; 2004, Chambers, Stuart; Johnston, Robert (Operation Management) $\{$ FT_prentice Hall $\}$. 